Menschen durch den Menschen, das Absterben des Staates, Züge, die sich als höchst unbequeme Vergleichsmaßstäbe erweisen, wenn die wirklichen Errungenschaften der kommunistischen Staaten daran gemessen werden.

Darüber hinaus betrachtete Löwenthal es als ein Problem, dass die kommunistische Ideologie das sogenannte Führerprinzip verwarf und >demokratisch erscheinen wollte. Aus diesem Grund waren sowohl die »Wirklichkeit der Parteidiktatur« als auch die "zentralistische Disziplin« schwerer zu rechtfertigen. Da aber »blinder Glaube« offiziell nicht gefordert werden durfte, musste diese Rechtfertigung in rationaler Form erfolgen. Eben dieses »ständige Bedürfnis«, so Löwenthal, versucht

den Schein rationaler Argumentation aufrechtzuerhalten [...], bedingt die stärkere Ausarbeitung der Ideologie gegenüber dem »irrationalistischen« Totalitarismus der Rechten, und gibt ihrer immer neuen Auslegung solche Bedeutung für den Zusammenhalt des Parteiregimes. Auf Grund der Fiktion von Demokratie und Rationalität hängt die Moral der Parteikader davon ab, daß der Anschein ideologischer Konsequenz aufrechterhalten wird. ${ }^{795}$

\title{
3.5 Analytisches Resümee
}

1. In dem hier zugrunde liegenden Untersuchungszeitraum besaßen die eingangs angesprochenen totalitarismustheoretischen Studien für die Thematisierung, Beschreibung und Analyse der beiden totalitären Herrschaftssysteme des Nationalsozialismus und des Sowjetkommunismus in der Zeitschrift Der Monat eine zentrale Bedeutung. Einerseits wurden drei Kapitel aus der 1951 in den Vereinigten Staaten erschienenen Studie The Origins of Totalitarianism von Arendt sowie das Eingangs- und Schlusskapitel aus dem 1952 veröffentlichten Buch The Origins of Totalitarian Democracy von Talmon abgedruckt. Andererseits spielten die entscheidenden analytischen Definitionsmerkmale bzw. Erklärungsansätze der einzelnen Totalitarismusstudien explizit oder implizit eine einflussreiche Rolle. Das gilt besonders für die analytischen Definitionsmerkmale von Arendt und für den von Eric Voegelin in seiner 1938 erschienenen Studie ins Leben gerufenen Forschungsansatz der "politischen Religionen«, aber auch für zentrale Kategorien aus der von Cohn 1957 herausgegebenen Studie The Pursuit of the Millennium. Zudem kann der Einfluss einzelner Merkmale des von Friedrich in seiner Untersuchung Totalitäre Diktatur vorgelegten »Sechs-Punkte-Kataloges« festgestellt werden; indes erfolgte in keinem Beitrag der Bezug auf sämtliche sechs analytischen Definitionsmerkmale und auf den interdepentenden Wirkungszusammenhang, d. h. auf die Tatsache, dass die einzelnen Merkmale für Friedrich nur gemeinsam ein Regierungssystem als totalitäre Diktatur kennzeichneten. Keinen ausdrücklichen Stellenwert besaß die 1956 erschienene Studie The Permanent Purge. Politics in Soviet Totalitarianism von Brzezinski, in der er, vor dem Hintergrund des Anspruchs des Parteiregimes, eine totale gesellschaftliche Revolution durchzuführen, letztlich die "permanente Säuberung« als zentrales Kriterium für das totalitäre System bestimmte.

Das Werk über die Ursprünge der »totalitären Demokratie« von Talmon bezog sich mit Blick auf die vergleichende Analyse des Nationalsozialismus und des Sowjetkom- 
munismus ausdrücklich ausschließlich auf den »linken« Totalitarismus. Erstens kam in der Einleitung zu seiner Untersuchung, die der Monat im Mai 1952 (H. 44) abdruckte, explizit zum Ausdruck, dass Talmon den Begriff der totalitären Demokratie nur auf den »Totalitarismus der Linken« bezog, weil er nämlich auf den »Totalitarismus der Rechten« nicht angewandt werden konnte. Zweitens bezogen sich die einleitenden Worte der Zeitschriftenredaktion zu dessen Veröffentlichung expressis verbis auf die sowjetische Besatzungszone in Ostdeutschland und deshalb auf den Stalinismus.

Die Theorie der »Politischen Religion« Voegelins hatte in zahlreichen Beiträgen im Monat einen starken Einfluss bei der Auseinandersetzung mit dem Totalitarismusphänomen. Im Grunde genommen fand sich sein ideengeschichtliche Erklärungsansatz für das Auftauchen der modernen politischen Massenbewegungen in Deutschland und der Sowjetunion (sowie in Italien) von Beginn an in der Zeitschrift wieder, wonach diese als Ausdruck einer fundamentalen Krise der westlichen Zivilisation zu verstehen seien, die wiederum - vor dem Hintergrund der Französischen Revolution und der Ideen der Aufklärung - aus der Säkularisierung des Geistes, d. h. der Trennung des »weltlichen Geistes« von seinen religiösen Wurzeln, resultierte. In diesem Zusammenhang wurde sehr wohl beleuchtet, dass das nationalsozialistische und das sowjetkommunistische Herrschaftsregime einerseits als das Ergebnis einer innerweltlichen Religion aufzufassen waren, die das Kollektiv (die Menschheit, das Volk, die Klasse, die Rasse oder den Staat) als Realissimum erlebten, nämlich als »Abfall von Gott«, sprich als Abfall von der »überweltlichen Religion« des Christentums, sodass beispielsweise auch der Nationalismus als »Ersatzreligion« (Kohn) aufgefasst wurde. Anderseits wurde auch zur Sprache gebracht, dass die beiden Regime angesichts der »Verlassenheit des Menschen« vorhandene religiöse Bedürfnisse der Individuen ausnutzten und im Kontext der staatlichen Politik gewissermaßen Kompensation boten. Genauer gesagt: Bei der Berücksichtigung der »Politischen Religion« zur Erklärung des Totalitarismusphänomens rückten auf einer abstrakten Ebene sehr wohl die komplexen politisch-religiösen, philosophischen, wissenschaftlichen und gesellschaftsgeschichtlichen Voraussetzungen für die staatliche Machteroberung der nationalsozialistischen und sowjetkommunistischen Massenbewegungen in den Fokus des Interesses. In diesem Kontext wurden auch die mentalen und (sozial-)psychologischen Aspekte für die Anfälligkeit der einzelnen sogenannten verlassenen und ängstlichen Menschen in der modernen kapitalistischen Industriegesellschaft nach dem »Abfall« von Gott angesprochen. Insofern wurde hierbei auch generell zur Sprache gebracht, dass es im Nationalsozialismus und im Sowjetkommunismus einen messianischen Führerkult bzw. -glauben gab, dass Hitler und Stalin (bzw. Lenin) als gottähnliche Herrscher betrachtet wurden, in die die Massen ihre heilsgeschichtlichen Erlösungsvorstellungen hineinprojizierten.

Aber auch die entscheidenden Aussagen aus der mit Blick auf die politisch-religiösen Massenbewegungen des Mittelalters und der Reformationszeit geschriebenen Studie von Cohn spielten durchaus eine Rolle bei der Beschreibung und Analyse des Nationalsozialismus und des Sowjetkommunismus. Insbesondere anhand des mit dem Augenmerk auf den Nachstalinismus geschriebenen Beitrages Ideologie und Realpolitik. Glauben die Kreml-Machthaber an Dogmen? (H.117) von Löwenthal konnte aufgezeigt werden, dass beim ausdrücklichen Vergleich des nationalsozialistischen und (sowjet-)kommunistischen Herrschaftssystems die Kategorien und (Untersuchungs-) Ergebnisse von Cohn auftauchten; allerdings erfolgte hierbei kein ausdrücklicher 
Verweis auf die Studie. Löwenthal hob nämlich für beide totalitären Regime und ihre unterschiedlichen Ideologien explizit hervor, dass Erwartungen eines »letzten Kampfes« und chiliastische Vorstellungen von einem Tausendjährigen Reich eine zentrale Rolle spielten, um eine »konfliktfreie Gesellschaft« entstehen zu lassen. Wie Cohn stellte auch Löwenthal fest, dass zum Erreichen ebendieses Zieles, der Kampf respektive die Säuberung der Welt durch die Ausrottung der »immer gleichen Feinde« (Löwenthal), d.h. der »Repräsentanten der Verderbnis« (Cohn), ein entscheidendes (Handlungs-)Motiv darstellte. Auf der einen Seite waren es »die Juden« bzw. das »Weltjudentum«, auf der anderen Seite die »Großen« bzw. »die Bourgeoisie« oder der »Monopolkapitalismus«, die für sämtliche gesellschaftlichen Missstände verantwortlich gemacht wurden. Somit tauchte beispielsweise die Figur des Teufels, die früher als Sündenbock für gesellschaftliche Missstände diente, in säkularisierter Form in den totalitären Ideologien des deutschen Nationalsozialismus und des sowjetischen Kommunismus wieder auf.

Während das Konzept der »Politischen Religion« für den gesamten hier zugrunde liegenden Untersuchungszeitraum bei der vergleichenden Beschreibung und Analyse des Nationalsozialismus und des Sowjetkommunismus herangezogen wurde - in diesem Zusammenhang wurden auch die ideengeschichtlichen und facettenreichen generellen gesellschaftsgeschichtlichen Voraussetzungen für die »Machtergreifung der Bolschewiki und der Nationalsozialisten thematisiert, ohne allerdings die politischen Bedingungen in den Blick zu nehmen -, besaßen die analytischen Kategorien aus Arendts Totalitarismusstudie beim Vergleich der beiden totalitären Herrschaftssysteme ausschließlich für den Zeitraum bis zum Tod Stalins eine zentrale Bedeutung. Genauer gesagt: Von Oktober 1948 bis zum Tod des sowjetischen Diktators, also bis zum Ende des Stalinismus, hatten sowohl die 1951 erschienene, zum Standardwerk der Totalitarismustheorie avancierte Untersuchung The Origins of Totalitarianism von Arendt als auch ihre entscheidenden analytischen Definitionsmerkmale für die »neue Staatsform« beim Vergleich des Nationalsozialismus und des Stalinismus im Monat einen herausragenden Stellenwert. Mit der Veröffentlichung des Beitrages Der imperialistische Charakter. Eine psychologisch-soziologische Studie wurde zum einen auf einer allgemeinen Ebene indirekt ein Element der Vorgeschichte für das Entstehen der totalitären Regime ins Licht gerückt; wobei sich die Ausführungen ausschließlich auf den englischen Imperialismus bezogen und sich vor allem auf die sowjetische Geschichte sicherlich nur unter Abstraktion der tatsächlichen historischen Erscheinungsform übertragen ließen. Zum anderen enthielten die beiden anderen Auszüge aus ihrem Buch, d.h. die Kapitel »Totalitäre Propaganda« und »Die Geheimpolizei«, substanzielle Einsichten für die vergleichende Analyse des Nationalsozialismus und des Stalinismus.

Zudem beriefen sich in diesem Zeitraum nicht nur einige Autoren des Monat ausdrücklich auf Arendt, sondern in zahlreichen veröffentlichten Beiträgen wurde auch das Erkenntnispotenzial ihrer Totalitarismusstudie be- und ausgenutzt, um die totalitären Regime aus Deutschland und der Sowjetunion zu beschreiben und zu analysieren sowie das spezifische Wesen und Prinzip des Totalitarismus zu bestimmen. Denn die analytischen Definitionsmerkmale für die neuartigen totalitären Herrschaftssysteme, die Arendt im Nationalsozialismus ab dem Jahre 1938 und im Stalinismus von 1930 bis 1953 historisch verwirklicht sah, wurden insbesondere in dem genannten Zeitraum von zahlreichen Autoren im Monat verwendet, auch dann, wenn sie sich nicht 
unbedingt ausdrücklich auf Arendt beriefen. Insofern lässt sich der Einfluss ihrer Totalitarismusstudie belegen, denn einerseits koinzidierte das von Arendt gezeichnete Bild der totalitären Herrschaft mit dem, welches im Grunde genommen in toto beim Vergleich des Nationalsozialismus mit dem Stalinismus im Monat verwandt wurde, um das Wesen der beiden Regime zu charakterisieren, andererseits gebrauchten $\mathrm{Au}$ toren in ihren Beiträgen zumindest einzelne Kategorien der arendtschen analytischen Definitionsmerkmale.

Vor dem Hintergrund der einzelnen analytischen Kategorien der neuartigen "totalen Organisation«, der »totalitären Propaganda« sowie von »Ideologie« und (permanenten) »Terror « spielte nicht zuletzt die arendtsche Erkenntnis in zahlreichen Beiträgen eine entscheidende Rolle, wonach vor allem die enge Affinität zwischen Ideologie und Terror den spezifischen totalitären Charakter des nationalsozialistischen und stalinistischen Herrschaftssystems bestimmte und die unter anderem auch die differentia spezifica sowohl zu traditionellen als auch zeitgenössischen (prä-)faschistischen oder diktatorialen Herrschaftssystemen ausmachte. Denn in den beiden »Weltanschauungsregimen« aus Deutschland und der Sowjetunion nahmen die Akteure die ideologischen Aussagen für »bare Münze« und machten ernst damit, sie durch (permanenten) Terror in Herrschaftswirklichkeit umzusetzen. Analog zu Arendt bedeutete dies für zahlreiche Autoren des Monat, dass die Ideologien der beiden Regime dazu dienten, die radikale Veränderung der Gesellschaft im Hinblick auf einen »Heilszustand « anzustreben: In der kommunistischen Ideologie stand die Vorstellung von »absterbenden Klassen « im Zentrum, in der nationalsozialistischen die von »parasitären Rassen«. Das totalitäre Moment der Verbindung von »Ideologie und Terror« ergab sich für diesen Personenkreis daraus, dass der Feindstatus dieser Menschengruppen aus den ideologisch vorgegebenen »objektiven Merkmalen« deduziert wurde, sodass die gewalttätige Ausschaltung der jeweiligen »Feindgruppen« als notwendige geschichtliche Handlung aufgefasst wurde. Auf diesen Aspekt gingen beispielsweise zwei Autoren ausdrücklich ein, die hier exemplarisch genannt werden: zum einen Hans Kudszus in seinen Ausführungen zu dem arendtschen Kapitel »Ideologie und Terror « in der Rezension der Festschrift für Karl Jaspers (H. 56); zum anderen Lasky, dessen gesamter Beitrag Die Moskauer Geständnisse. Gedanken über Ketzerei und Opposition im totalen Staat (H.36) von der arendtschen Argumentationslogik inspiriert war und die sich auch in seiner vergleichenden Analyse der beiden Regime sowie der nationalsozialistischen und stalinistischen Konzentrationslager niederschlug.

Bei allen inhaltlichen Unterschieden bei der Thematisierung des facettenreichen Totalitarismusphänomens und beim Vergleich des Nationalsozialismus und des Stalinismus kann letzten Endes festgestellt werden, dass im Monat ein Bild des totalitären Systems gezeichnet wurde, in dem eine kleine (kriminelle) Führungsgruppe bzw. »kleine Verbrecherbande« durch die spezifische Methode des Terrors, ausgeübt durch den Apparat der Geheimpolizei, ihre totale Herrschaft sicherte. Zudem wurde immer wieder festgestellt, dass die Exponenten der beiden totalitären Systeme ihre Herrschaft dadurch sicherten, dass sie es schafften, ihren Einfluss bis in die Privatsphäre des Menschen geltend zu machen. Nicht zuletzt mittels der Totalitarismusstudie und der historisch-politischen Erkenntnisse von Arendt kam immer wieder zum Ausdruck, dass sowohl der Nationalsozialismus als auch der Stalinismus allumfassende, alles verschlingende Herrschaftssysteme waren, in denen durch einen allgegenwärtigen Terror die gesamte Gesellschaft atomisiert war, in denen die Angst allgegenwärtig war 
und es keinen noch so kleinen Freiraum geben sollte und in denen zudem die politische Ideologie nicht in irgendeiner Weise präsent war; ebendiese lückenlose Herrschaftspräsenz war bekanntlich auch ein zentraler Gedanke in dem von Orwell geschriebenen und im Monat abgedruckten Buch $1984 .{ }^{796}$

In diesem Zusammenhang wurde nicht nur für Arendt das System der Konzentrationslager zum Inbegriff der neuartigen totalitären Herrschaftsform, sprich für den Nationalsozialismus und den Sowjetkommunismus stalinistischen Typs. Im Grunde genommen herrschte unter den Autoren des Monat Konsens darüber, dass die Konzentrationslager des sowjetischen Staates und die Konzentrations- und Vernichtungslager des NS-Staates als - mit Arendt gesprochen - „Laboratorien« für ihren genuinen Anspruch dienten, dass Menschen total beherrschbar waren. Daraus folgte auch die sozusagen analytische Erkenntnis, dass sowohl für das nationalsozialistische als auch für das stalinistische Herrschaftsregime, deren Wesen der (permanente) politische Terror war, das System der Konzentrationslager zentraler Bestandteil war und dass sie ohne die Lager letzten Endes nicht existieren konnten.

Im Hinblick auf die Frage, welches Totalitarismusmodell der Monat favorisierte, kann festgestellt werden, dass sich für den hier zugrunde liegenden Untersuchungszeitraum die Einflüsse sowohl von Voegelins Studie Die politischen Religionen als auch der in den 1950er-Jahren erschienenen unterschiedlichen totalitarismustheoretischen Studien - mit Ausnahme der von Brzezinski - aufzeigen lassen; wenn auch in unterschiedlichem Maße. Mit Blick auf den »Sechs-Punkte-Katalog« von Friedrich muss indes konzediert werden, dass nur einzelne Merkmale seines Totalitarismusmodells von Bedeutung waren und infolgedessen nicht seine Auffassung eines interdepentenden Wirkungszusammenhanges der sechs analytischen Definitionsmerkmale. Zudem kann festgestellt werden, dass das Konzept der »Politischen Religion« (Voegelin, Cohn) für die vergleichende Analyse des Nationalsozialismus und des Stalinismus (und Nachstalinismus) im Grunde genommen durchgehend eine wichtige Rolle spielten, wie auch die Studie von Talmon.

Indes: Für den Zeitraum bis zum Tod Stalins besaß vor allem die Studie von Arendt im Allgemeinen und ihre analytischen Definitionsmerkmale im Besonderen eine überragende Bedeutung. Für den Zeitraum danach kann festgestellt werden, dass fortan Arendts Totalitarismustheorie im Rahmen der ausdrücklichen vergleichenden Analyse des Nationalsozialismus und des Nachstalinismus keine Rolle mehr spielte, zumal nur noch in einem einzigen Beitrag die substanzielle Gegenüberstellung der beiden Systeme im Mittelpunkt stand: Hierbei handelte es sich um die Veröffentlichung Die Hölle aufErden. Despotie im zwanzigsten Jahrhundert von Löwenthal, die im Juniheft des

796 Arthur [M.] Schlesinger jr., Chancen eines liberalen Kommunismus. Gedanken nach einer Reise durch Osteuropa, in: Der Monat 12 (1960), H. 137, S. 39-52, räumte in seinem Beitrag, der sich ausdrücklich mit dem Wandlungsprozess des sowjetischen Herrschaftssystems unter Chruschtschow auseinandersetzte, aufS. 52 ein, dass in einer Welt, die nach dem Ende des Zweiten Weltkrieges von den »Schrecken der nazistischen Konzentrationslager« erfuhr und »sich nun furchtgebannt dem Fanatismus des stalinistischen Kommunismus gegenübergestellt sah«, es nahelag, den »Totalitarismus als allumfassenden, alles verschlingenden, unverfälschten Begriff der absoluten Macht anzusehen«. Und weiter hieß es bei Schlesinger jr., dass Orwell und Arendt ebendieser »Vorstellung in denkwürdiger Form Ausdruck verliehen« hatten »und für einen Augenblick ließen wir uns alle von dem Gedanken bestechen, daß die Umwandlung einer gewöhnlichen Gesellschaft zu einer totalitären auch die Umwertung aller gewöhnlichen menschlichen Motive mit sich bringe«. 
Jahres 1957 (H. 105) erschien; dasselbe galt für den Vergleich der deutschen und sowjetischen Konzentrationslager, der ebenfalls nur bis zum Tode Stalins vorgenommen wurde. Darüber hinaus wurden der deutsche und der sowjetische Totalitarismus ausschließlich im Bereich der »Politischen Religion « ausdrücklich miteinander verglichen, und das in einem Beitrag von Löwenthal, der sich indes in erster Linie mit dem Nachstalinismus auseinandersetzte: Hierbei handelte es sich um den Beitrag Ideologie und Realpolitik. Glauben die Kreml-Machthaber an Dogmen? vom Juni 1958 (H. 117).

2. Im Hinblick auf die Frage, wie letzten Endes der ausdrückliche Vergleich des nationalsozialistischen mit dem sowjetkommunistischen Herrschaftssystem - insbesondere in der stalinistischen Phase - ausfiel, kann grundsätzlich festgestellt werden, dass vor dem Hintergrund des Einflusses der unterschiedlichen Totalitarismustheorien die historisch-politische und theoretische Gegenüberstellung der beiden Regime von Anbeginn im Monat eine Rolle spielte. In diesem Zusammenhang wurden auch ganz allgemein die gesellschaftsgeschichtlichen Voraussetzungen für das Entstehen der beiden Massenbewegungen sowie der staatlichen Machteroberung durch die Bolschewiki und der Nazis thematisiert; die beispielsweise von Kohn (Geschichte und Politik, H. 45) im Entstehen der nationalistischen und sozialistischen Massenbewegungen nach der 1848er-Revolution zu sehen waren. Gemeinhin wurde allerdings der Ausbruch des Ersten Weltkrieges als die entscheidende Zäsur angesehen, und für Löwenthal (Die Hölle aufErden. Despotien im zwanzigsten Jahrhundert, H. 105) war die "materielle Not der Massen«, also die politischen, wirtschaftlichen und sozialen Probleme der Menschen in Russland und Deutschland infolge der rasanten industriellen Entwicklung, insbesondere seit dem Ersten Weltkrieg die entscheidende Erklärung.

Ergänzend $\mathrm{zu}$ den durch die Totalitarismustheorien zutage geförderten Erkenntnisse und zu dem in diesem Zusammenhang gezeichneten Bild des Nationalsozialismus und des Stalinismus kann - bei allen unterschiedlichen Perspektiven und Schwerpunkten - in Bezug auf den ausdrücklichen Vergleich der totalitären Staaten aus Deutschland und der Sowjetunion zusammenfassend festgestellt werden, dass bei der vergleichenden Analyse der beiden politischen Systeme in den Beiträgen der Autoren Folgendes zum Ausdruck kam:

a. Dass die vergleichende Analyse des nationalsozialistischen und des sowjetkommunistischen Herrschaftssystems speziell unter Stalin durch eine totalitarismustheoretische Brille geschärft war.

b. Dass es sich beim Nationalsozialismus und Stalinismus um neuartige, genuin antidemokratische Einparteienregime handelte, deren totalitärer Charakter sich nicht zuletzt aufgrund der von ihnen begangenen beispiellosen Massenverbrechen erklärte.

c. Dass die nationalsozialistischen und stalinistischen Herrschaftssysteme zwar nicht identisch waren, sich ihre genuine Verwandtschaft indes daraus ergab, dass das Wesen der beiden Regime politischer Terror war, der aus systemimmanenten Gründen einen permanenten Charakter besaß und zu einer "permanenten Institution « (Borkenau) figurierte. Anders gesagt: Für beide Regime galt, dass sich in ihnen die politische Gewalt vergrößerte, intensivierte und dynamisierte. In diesem Zusammenhang war auch der Konnex zwischen Ideologie und Terror entscheidend: Der Terror des Nationalsozialismus richtete sich aus der rassistisch-antisemitischen Weltanschauung gegen die (europäischen) Juden, der »rote 
Terror« des sowjetischen Kommunismus richtete sich aus der spezifischen Ideologie gegen sogenannte Konterrevolutionäre und den »Klassenfeind«.

d. Dass das »Hitler-Regime« und das »Stalin-Regime« aus systemimmanenten Gründen innere und äußere Feinde benötigten. Im Nationalsozialismus war es das »internationale Judentum«, im sowjetischen Kommunismus die »Bourgeoisie« schlechthin. Ohne ebendiese Feindobsession konnten im Grunde genommen beide Systeme nicht existieren, da sie vor dem Hintergrund der Herrschaftssicherung in erster Linie dazu dienten, die gesamte Gesellschaft »unter Spannung«(Schlesinger jr.) zu halten und sie nicht zur Ruhe kommen zu lassen.

e. Dass sowohl der nationalsozialistische als auch der sowjetkommunistische, genauer der stalinistische Terror, insbesondere zu dem Zeitpunkt in Gang kam, als die gewalttätige Ausschaltung der tatsächlichen Opposition der Regime erfolgreich abgeschlossen war.

f. Dass die deutschen und sowjetischen Konzentrationslager fester Bestandteil der beiden totalitären Regime waren, oder anders gesagt, dass das System der Konzentrationslager zum Synonym für die Terrorregime avancierte. In diesem $\mathrm{Zu}$ sammenhang wurde von Arendt angesprochen, dass sie neben der genuin politischen Bedeutung auch eine implizit ökonomische Funktion besaßen: nämlich die Finanzierung des »Himmler-Apparates « und des NKWD.

g. Dass es sich beim Nationalsozialismus und beim Stalinismus um Regime handelte, in denen eine »kleine Gruppe verbrecherischer Männer« (Churchill) mit dem neu geschaffenen Instrument des Geheimpolizeiapparates ihre totale Herrschaft über die gesamte Gesellschaft sicherten.

h. Dass sich der totalitäre Charakter des deutschen und sowjetischen Herrschaftssystems auch dadurch erklärte, dass sie keine »Insel der Freiheit« (Friedrich) duldeten und mithin organisierter politischer und militärischer Widerstand wenn nicht unmöglich, so doch zumindest die Ausnahme darstellte und nicht vorhersehbar war.

i. Dass es zu den charakteristischen Eigenschaften der beiden Regime gehörte, dass sie grundsätzlich mit den neuartigen Herrschaftsmethoden der »Propaganda und Lüge« operierten, wie es sich während des Zweiten Weltkrieges exemplarisch im »Fall Katyn« gezeigt hatte.

j. Dass sich die Verwandtschaft der beiden Systeme auch aus der Tatsache ergab, dass sie in Anbetracht jeweils unterschiedlicher Ideologien die Weltherrschaft anstrebten.

k. Dass im Hitler-Stalin-Pakt die politische Zusammenarbeit des Nationalsozialismus und des Sowjetkommunismus im Verständnis der westlichen Demokratien seinen Höhepunkt erreichte. Ohne den deutsch-sowjetischen Nichtangriffspakt, der über das zwischenstaatliche Nichtangriffsversprechen hinaus eine absolute Neutralität festlegte, sowie das »Geheime Zusatzprotokoll«, das zwischen den beiden imperialen Mächten die Vereinbarung enthielt, den polnischen Staat in jeweils festgelegte territoriale Bereiche untereinander aufzuteilen, wäre der Ausbruch des Zweiten Weltkrieges nicht möglich gewesen. Der Hitler-Stalin-Pakt wurde nach dem Dafürhalten des Monat zum Synonym für existenzielle Gefährdungen, die den westlichen Demokratien durch den Totalitarismus schlechthin drohen.

1. Dass nach dem Zusammenbruch des `Dritten Reiches für die westlichen Demokratien eine erneute existenzielle (Kriegs-)Gefahr existierte, weil der von Moskau 
beherrschte internationale Kommunismus qua Definition die Zerstörung der kapitalistischen Staaten auf seine »Fahnen« geschrieben hatte. Indes: Auch wenn es sich um expansive Systeme handelte, unterschied sich die nationalsozialistische und sowjetkommunistische Außenpolitik nicht zuletzt im Hinblick auf die verschiedenen diplomatischen Methoden: Hitler kannte nur die gewaltsame Methode, Stalin wiederum agierte taktisch und strategisch vorsichtiger und cleverer. Ein weiterer Unterschied wurde dem Umstand zugeschrieben, dass der Nationalsozialismus neben politisch-ideologischen Motiven den Krieg auch aus ökonomischen Gründen mit dem Ziel geführt hatte, »Lebensraum« in Osteuropa zu gewinnen, um nicht zuletzt an dringend benötigte Rohstoffe zu gelangen.

m. Dass Planwirtschaft, zumal in staatskapitalistischer Form, letzten Endes mit Demokratie und einem parlamentarischen »Vielparteiensystem « a priori nicht möglich war. Mit anderen Worten: Jedwede Abschaffung des Privateigentums und die Einführung einer starken Zentralisierung im wirtschaftlichen Bereich respektive des sogenannten demokratischen Kollektivismus barg eine totalitäre Gefahr, weil damit auch die Tendenz zur politischen Zentralisierung verbunden war.

n. Dass die sozialpsychologischen bzw. mentalen Dispositionen der Anhänger der totalitären revolutionären Bewegungen im Grunde genommen identisch waren, sodass einzelne Akteure nicht zuletzt aufgrund ihres antibürgerlichen Ressentiments und des sozialrevolutionären Motivs ohne Weiteres vom Nationalsozialismus zum Kommunismus wechseln konnten und vice versa.

3. Im Hinblick auf die zentralen Fragen in diesem Teil der vorliegenden Untersuchung, inwiefern sich der Monat bei der vergleichenden Analyse des nationalsozialistischen und des sowjetkommunistischen Herrschaftssystem im Fahrwasser der zeitgenössischen politisch-wissenschaftlichen Sowjetforschung der 1950er-Jahre befand und in diesem Kontext auch eine »normative Funktion« (Martin Jänicke) besaß, kann zusammenfassend festgestellt werden:

a. Wie in der 1956 erschienenen totalitarismustheoretischen Studie The Permanent Purge. Politics in Soviet Totalitarianism von Brzezinski erschien auch im Monat der sowjetische Totalitarismus als das am weitesten fortgeschrittene totalitäre Herrschaftssystem und infolgedessen am Ende als der eigentliche Fall eines totalitären Herrschaftsmodells.

b. In Anbetracht der Grundannahme, dass die sowjetisch-kommunistische Parteidiktatur, insbesondere in der stalinistischen Phase, als eigentlicher Modellfall totalitärer Herrschaft aufzufassen war, verzichtete der Monat bei der vergleichenden Analyse des Nationalsozialismus mit dem Sowjetkommunismus (Stalinismus/Nachstalinismus) darauf, die zeitgenössischen Forschungsergebnisse zur Geschichte des >Dritten Reiches< heranzuziehen. Insofern erfolgte der Vergleich des nationalsozialistischen mit dem sowjetkommunistischen Herrschaftssystem, speziell in Gestalt des Stalinismus, letzten Endes nicht auf der Grundlage und der Kenntnis der zeitgenössischen Forschungsliteratur zur Geschichte des >Dritten Reiches .

c. Die normative Funktion bestand darin, dass es beim Vergleich des nationalsozialistischen und sowjetkommunistischen Herrschaftssystems insbesondere unter Stalin primär um ein politisches Urteil ging, dessen zentrales Ziel in den Jahren 
des Kalten Krieges in Westdeutschland die grundsätzliche Delegitimation des sowjetischen Kommunismus, der von Moskau dominierten osteuropäischen Satellitenstaaten sowie des SED-Regimes war.

Wie nämlich aufgrund der zahlreichen Veröffentlichungen gezeigt werde konnte, kam im Monat immer wieder zum Ausdruck, dass schlussendlich aus der Sicht der westlichen Demokratien nicht das "Hitler-Regime«, sondern das sowjetische Regime insbesondere in der stalinistischen Phase der gefährlichere Gegner war und als eigentlicher Totalitarismus aufzufassen sei. Zur Begründung, dass für die westlichen Demokratien der sowjetische Kommunismus die größere Gefahr darstellte, führte beispielsweise Hook an, dass im Unterschied zum »Hitler-Regime« der sowjetische Kommunismus in den demokratischen Staaten über eine »viel stärkere Fünfte Kolonne«verfügte. Und Wilhelm Röpke begründete die unterschiedliche Gefahr mit Blick auf die "geistigen Grundlagen des Abendlandes« damit, dass der Bolschewismus einen »universellen und rationalistischen Charakter « besaß (und in diesem Kontext ebenfalls über eine »Fünfte Kolonne« verfügte) und dass hierzu wiederum der Nationalsozialismus nur über eine »wirre Theorie« des »Herrenvolkes« verfügte, die noch nicht einmal einen »Hottentotten« überzeugen konnte.

Dieselbe tendenziöse Auffassung ließ sich auch in dem Beitrag über den Juni-Aufstand 1953 (H. 59) in Ostdeutschland von Salter feststellen, wo der Autor die Auffassung vertrat, dass sich letzten Endes das vom NKWD errichtete Herrschaftsregime im Vergleich zur »Perfektion der Gestapo« als überlegen erwies, weil es in diesem Zusammenhang ausdrücklich die Erfahrungen aus der Sowjetunion mit dem »Hitler-Reich« kombinieren konnte.

Auf der anderen Seite vertrat Schlesinger jr. in einem der zentralen Beiträge im Monat (Politik im Zeitalter der Angst, H.16 und 19) zum Totalitarismusphänomen in der ersten Hälfte des 20. Jahrhunderts im Allgemeinen und zur vergleichenden Beschreibung und Analyse der Geschichte des >Dritten Reiches` und des Sowjetkommunismus im Besonderen die Auffassung, dass der Nationalsozialismus letzten Endes einen »unvollkommenen Totalitarismus« verkörperte. Zur Begründung führte er bekanntlich an, dass der sowjetische Staat im Gegensatz zum »Hitler-Staat« die Wirtschaftsstruktur des Privateigentums radikal verändert hatte, d. h. nach der Oktoberrevolution von 1917 die »besitzende Klasse« systematisch enteignete und mithin die bis dahin »herrschenden Bevölkerungsteile« liquidierte. Da der Nationalsozialismus im Gegensatz zum Sowjetkommunismus letzten Endes das Privateigentum der (staatskapitalistischen) Wirtschaft nicht antastete, führte dies dazu, dass in Deutschland eben nicht die »Einheit von Staat und Gesellschaft« verwirklicht wurde und mithin das NS-Regime insgesamt "verwundbarer« war als das sowjetische Regime (sowohl unter Lenin als auch unter Stalin) - was für Schlesinger jr. im »Putschversuch« vom 20. Juli exemplarisch unter Beweis gestellt wurde.

Schlesingers Beitrag Politik im Zeitalter der Angst zählte zudem zu einem der wenigen Veröffentlichungen im Bereich der vergleichenden Beschreibung und Analyse der Geschichte des >Dritten Reiches« und des sowjetischen Kommunismus, in dem ausdrücklich die jeweilige Forschungsliteratur herangezogen wurde und die Genesis der staatlichen Machteroberung sowie -konsolidierung der beiden totalitären Bewegungen in den Fokus gerückt wurde. Auch wenn in diesem Zusammenhang sicherlich ein facettenreiches vergleichendes Bild der totalitären Herrschaftssysteme und vom We- 
sen des Totalitarismus gezeichnet wurde, gewann Schlesinger jr. indes seine Ergebnisse primär am Material des Stalinismus, ohne sie mit den grundsätzlichen zeitgenössischen Forschungsergebnissen zur Geschichte des Nationalsozialismus zu prüfen.

Auch bei der vergleichenden Beschreibung und Analyse der deutschen und sowjetischen Konzentrationslager wurden die Ergebnisse ebenfalls primär am Material des Stalinismus gewonnen. Mit Ausnahme der kurzen Ausführungen von Arendt zur Rolle der Lager und der Sklavenarbeit für die Finanzierung des SS-Apparates und des NKWD diente das nationalsozialistische System der "Konzentrations- und Vernichtungslager (Arendt) in erster Linie als Negativfolie für die stalinistischen Lager. Insofern wurden beim Vergleich der nationalsozialistischen und stalinistischen Lager auch die insbesondere seit dem Zusammenbruch des >Dritten Reiches zutage geförderten Erkenntnisse über das deutsche Lagersystem - beispielsweise durch die 13 Nürnberger Prozesse, einzelne Monografien im Allgemeinen und durch die Forschungsliteratur im Besonderen, oder auch dem Kapitel »Die Konzentrationslager « aus Arendts Totalitarismusstudie - nicht explizit herangezogen. Folglich stand beim Vergleich der deutschen und sowjetischen Lager in erster Linie die politisch-ideologische Bedeutung, d.h. der Terror, im Mittelpunkt. Daraus folgte besonders, dass die ökonomische Funktion oder ökonomische Aspekte der Konzentrationslager ausschließlich mit Blick auf das stalinistische System thematisiert und hervorgehoben wurde, sodass die zeitgenössischen NS-Forschungsergebnisse speziell zu den deutschen Konzentrationslagern bei der vergleichenden Beschreibung und Analyse nicht berücksichtigt wurden. Letztlich besaß der Lagervergleich der beiden totalitären Regime eine normative Funktion, mit dem das grundsätzliche politische Ziel verfolgte wurde, den sowjetischen Kommunismus zu delegitimieren.

Im Grunde genommen wurden spezifische zeitgenössische NS-Forschungsergebnisse neben den beiden Beiträgen Die Geheimpolizei und Totalitäre Propaganda von Arendt ${ }^{797}$ ausschließlich noch in zwei Veröffentlichungen ausdrücklich beim vergleichenden Blick auf das Totalitarismusphänomen deutscher und sowjetischer Provenienz herangezogen, genutzt und zur Sprache gebracht: zum einen im Kontext des »Röhm-Putsches« im oben genannten Schlesinger-jr.-Beitrag, zum anderen in dem ausführlich wiedergegebenen zweiteiligen Beitrag zum Hitler-Stalin-Pakt von A. Rossi (H.11 u. 12), um nämlich nicht zuletzt die politischen Ziele Hitlers im Zweiten Weltkrieg hervorzuheben. Gleichwohl zeigte sich vor allem in der ausführlichen Auseinandersetzung mit dem deutsch-sowjetischen Nichtangriffsvertrag und seinen historisch-politischen Implikationen, wie die zeitgenössischen Forschungsergebnisse zur Geschichte des >Dritten Reiches` genutzt wurden - hierbei wurden sogar Dokumente des Nürnberger Hauptkriegsverbrecherprozesses als Quellenmaterial herangezogen -, um sie für eine politische Untersuchung zu gebrauchen. Das heißt, hier ging es durch einen sowohl einseitigen als auch instrumentellen Gebrauch der zeitgenössischen Forschungsergebnisse zur Geschichte des >Dritten Reiches $<$ und einzelner Nürnberger Dokumente in der Hochphase des Kalten Krieges um ein primär politisches Urteil über den sowjetischen Staat unter Stalin und seiner aktiven Rolle beim HitlerStalin-Pakt. Auch die Untersuchung über den deutsch-sowjetischen Nichtangriffs-

797 Dass allerdings bei der Veröffentlichung der beiden Beiträge im Monat der umfangreiche Fußnotenapparat aus der Totalitarismusstudie von Arendt bis auf wenige Ausnahmen nicht mitabgedruckt wurde, wurde bereits oben erwähnt. 
vertrag besaß insbesondere eine normative Funktion mit dem Ziel der Delegitimation des zeitgenössischen sowjetischen Kommunismus. Beispielsweise kam nämlich in der Veröffentlichung zum Ausdruck:

a. Dass Stalin der eigentliche handelnde Akteur und Nutznießer des deutsch-sowjetischen Nichtangriffsvertrages war, und zwar durch die Tatsache, dass der »Kreml-Chef« immer wieder neue Forderungen stellte und im Grunde die Hauptverantwortung für den sogenannten Vertragsbruch trug.

b. Dass Hitler sich grundsätzlich als treuer Vertragspartner erwies, der im Grunde genommen mit dem Vertrag ausschließlich die Absicht verfolgte, im Rahmen der »Neuordnung Europas« den »Zweifronten-Krieg« zu vermeiden, um ausschließlich sein zentrales Ziel zu erreichen, Polen zu überfallen, um damit den benötigten »Lebensraum im Osten« zu gewinnen.

c. Dass erst die stalinsche Außenpolitik und seine speziellen politischen Forderungen gegenüber Deutschland zur kriegerischen Auseinandersetzung zwischen Nazi-Deutschland und der Sowjetunion führte.

d. Dass Stalin durch seine unmittelbare Politik im Kontext der Aufteilung Polens zwischen dem deutschen und sowjetischen Staat für die nationalsozialistischen Massenverbrechen an den Millionen sogenannten jüdischen Einwohnern innerhalb des »Generalgouvernement Polens « eine Mitverantwortung zu tragen hatte, weil er Hitler in Sachen der nationalsozialistischen »Polenpolitik« zum »Handeln gezwungen« hatte.

In diesem Zusammenhang war es zudem auffallend, dass die »kleine Studie« von Rossi ausschließlich das historisch-politische und diplomatische Geschehen bis November 1940 untersuchte; nicht zuletzt im Rahmen der Thematisierung der unmittelbaren Folgen nach dem Überfall der Wehrmacht auf Polen. Mithin kamen die in der Folge des Zweiten Weltkrieges in Gang gekommen deutschen Massenverbrechen ausschließlich mit Blick auf das polnische Territorium zur Sprache. Nicht berücksichtigt wurden infolgedessen die NS-Massenverbrechen an der sowjetischen Bevölkerung und speziell an den sowjetischen Juden, die nach dem Überfall auf die Sowjetunion am 1. September 1941 vor allem von den »Einsatzgruppen« begangen wurden und die beispielsweise durch die Nürnberger Prozessdokumente nicht nur bekannt waren, sondern auch bewiesen waren. Dass auch die Sowjetunion unter Stalin Opfer des vom nationalsozialistischen Herrschaftssystem ausgegangenen Zweiten Weltkrieges war, spielte im Zusammenhang des Vergleichs der beiden totalitären Herrschaftssysteme nicht nur bei Rossi keine Rolle. 